\title{
Relevance of serum interleukin-33 and ST2 levels and the natural course of chronic hepatitis $B$ virus infection
}

\author{
Shu-Ling Huan', Ji-Guang Zhao ${ }^{2}$, Zhen-Li Wang ${ }^{1}$, Shuai Gao ${ }^{1}$ and Kai Wang ${ }^{1,3^{*}}$
}

\begin{abstract}
Background: Interleukin-33 (IL-33) and ST2 have been demonstrated to be associated with liver damage. However, their potential value in hepatitis B virus (HBV) infection remains unknown. This study was designed to investigate the change of serum IL-33 and ST2 levels in the natural course of chronic HBV infection.

Methods: A total of 120 patients with chronic hepatitis B (CHB), 20 chronic hepatitis B virus carriers in immunotolerant phase and 28 healthy controls were enrolled in this study. All patients with CHB were divided into four groups according to their serum ALT levels. The serum levels of IL-33 and ST2 of all participants were determined by enzyme-linked immunosorbent assay, and compared between each two out of those six groups.

Results: No significant differences were found in serum levels of IL-33 and ST2 between the group of CHB with ALT 1-2 upper limit of normal and the healthy controls ( $P=0.354$ for IL-33 and $P=0.815$ for ST2). Other than that, there were significant differences when serum levels of IL-33 and ST2 were compared between any other two out of those six groups ( $P<0.05$, respectively). The overall correlation analysis indicated that changes of serum IL-33 and ST2 levels were positively associated with ALT levels in patients with chronic HBV infection ( $r s=0.879, P<0.001$ for IL-33 and $r s=0.923, P<0.001$ for ST2). No significant differences were found when the serum levels of ALT, IL-33 and ST2 were compared between patients with $\mathrm{HBeAg-positive} \mathrm{CHB}$ and HBeAg-negative CHB.
\end{abstract}

Conclusions: Our study revealed that the serum levels of IL-33 and ST2 varied in different courses of chronic hepatitis $B$ virus infection. The serum levels of IL-33 and ST2 elevated as serum ALT levels increased in patients with CHB. They might indicate liver damage for patients with CHB, just like ALT.

Keywords: Chronic hepatitis B, Interleukin-33(IL-33), ST2, Natural course, Enzyme-linked immunosorbent assay (ELISA)

\section{Background}

Interleukin-33 (IL-33), a new member of the IL-1 family, induced the production of pro-inflammatory and $\mathrm{T}$ helper-2 (Th2)-associated cytokines. ST2 was to weaken Th2 inflammatory responses as its receptor [1]. Hepatitis B virus (HBV) infection is still a major health problem worldwide. There are approximately 350 million people chronically infected with HBV globally, and they are at great risk of developing liver cirrhosis and hepatocellular carcinoma. About $5 \%$ of adulthood and over $90 \%$ of

\footnotetext{
* Correspondence: wangdoc876@126.com; wangdoc2010@163.com 'Department of Hepatology, Qilu Hospital of Shandong University, Jinan 250012, Shandong, China

${ }^{3}$ Institute of Hepatology, Shandong University, Wenhuaxi Road 107\#, Jinan 250012, Shandong, China

Full list of author information is available at the end of the article
}

infants and young children who are infected with HBV will evolve chronicity [2]. Many patients with chronic hepatitis $\mathrm{B}(\mathrm{CHB})$ ultimately progress to liver cirrhosis and hepatocellular carcinoma [3, 4]. Previous studies showed that the interaction of $\mathrm{HBV}$, hepatocytes and the host immune system determines the persistence of HBV infection and chronic inflammation [5]. The immune system was known to be suppressed by the viral infection and related hepatocyte injuries [6, 7]. Although experimental evidence suggested that antigen-specific Th1 immunity and pro-inflammatory cytokines played an important role in the HBV related liver injury and clearance of viruses $[8,9]$, serum IL-33 levels were associated with liver damage of patients with $\mathrm{CHB}[10]$, serum levels of IL-33 and soluble ST2 elevated in liver 
failure, which could be a sign of immune hyperactivation, and/or a mechanism to down-regulate inflammation [1]. Soluble ST2 may be useful to discern acute from chronic hepatic failure or to monitor the course and the severity of the disease. It is widely accepted that the adaptive immune responses play major roles in the clearance of HBV infection. However, the role of innate immunity during HBV infection appears not to be well understood, which can be attributed to the fact that the recruitment of patients in the very early, asymptomatic phase of HBV infection is very difficult $[11,12]$.

Remarkable progress had been made in the understanding of the natural history of chronic HBV infection. The natural course of chronic HBV infection was perceived as consisting of 4 phases: immune tolerance, immune clearance (HBeAg-positive chronic hepatitis B), inactive carrier state, and reactivation ( $\mathrm{HBeAg-negative}$ chronic hepatitis B) [13]. Although serum levels of IL-33 and ST2 were up-regulated in liver failure and $\mathrm{CHB}$, the relevance of serum levels of both molecules and patients with hepatitis B virus infection needed further research and serum alanine aminotransferase (ALT) activity is an important marker for liver damage in patients with $\mathrm{CHB}$. We therefore sought to investigate the serum measurements of IL-33 and ST2 in patients with chronic hepatitis B virus infection and study the relationship of IL-33 and ST2 with ALT and HBeAg. We examined the levels of serum IL-33, ST2 and ALT in patients with $\mathrm{CHB}$, chronic hepatitis B virus carriers in immunotolerant phase and healthy controls $(\mathrm{HC})$ so that we could determine whether IL-33 and ST2 had relevance with chronic HBV infection in our study.

\section{Methods}

\section{Patients}

A total of 120 patients with CHB, 20 chronic hepatitis B virus carriers in immunotolerant phase, and $28 \mathrm{HC}$ were successively conscribed at the outpatient and inpatient services in Qilu Hospital of Shandong University and Qingdao Municipal Infectious Disease Hospital from September 2012 through May 2014. The patients with $\mathrm{CHB}$ and chronic hepatitis B virus carriers in immunotolerant phase were defined according to the criteria of Asian-Pacific consensus statement on the management of chronic hepatitis B [14]. All patients with CHB were stratified into four groups according to their serum levels of ALT, including ALT1-2 upper limit of normal (ULN), ALT2-5ULN, ALT5-10ULN, ALT > 10ULN. The number of patients with $\mathrm{CHB}$ in each group was calculated based on the data obtained from preliminary experiment by using PASS 11 . Besides, all patients with $\mathrm{CHB}$ were divided into two groups of 65 patients with HBeAg-positive chronic hepatitis B and 55 patients with HBeAg-negative chronic hepatitis B according to their serum HBeAg. Patients with chronic HBV infection were confirmed positive for $\mathrm{HBsAg}, \mathrm{HBeAg}$ or $\mathrm{HBeAb}$ and detectable HBV virus for at least 12 months [13]. People who had comorbidities such as history of infection, hodiernal hepatitis A, C, D and E viruse infection, human immunodeficiency virus infection, autoimmune hepatitis, metabolic liver disease et al., were eliminated. All patients had not received any therapy of liver injury or been exposed to obvious liver damage compound.

Written informed consent was obtained from individual participants, and the study was approved by Ethical Committee of Qilu Hospital of Shandong University and Qingdao Municipal Infectious Disease Hospital.

\section{Laboratory assays}

Sera were extracted after blood samples were taken from all the participants and then stored at $-80^{\circ} \mathrm{C}$ till needed. The measurements of serum IL-33 and ST2 in all participants were determined by enzyme-linked immunosorbent assay (ELISA) using human IL-33 and ST2 ELISA Kits (Immunoway Biotechnology, USA) according to the manufacturer's instruction. The measurements of serum IL-33/ST2 in individual samples were calculated according to the standard curve established using the recombinant IL-33 and ST2 provided.

HBV-related HBsAg, HBsAb, HBcAb, HBeAg, and $\mathrm{HBeAb}$ were detected by an electro-chemiluminescence immunoassay using Roche kits (Roche Diagnostics, Germany) according to the manufacturer's instruction. The level of serum ALT was detected by Velocity method using big biochemistry automatic analyzer (Olympus 2700, Japan).

The serum HBVDNA levels were measured by nested reverse transcription polymerase chain reaction (PCR) quantitative assay using a luciferase quantitization detection kit, following the protocols (Roche Amplicor, Germany). The detection limit of viral DNA was 300 copies/ml [12, 15].

\section{Statistical analysis}

The data were expressed as the median (inter-quartile range). The differences between the groups were analyzed by Mann-Whitney $U$-test and overall correlation between ALT and IL-33, as well as ST2 were analyzed by Spearman's rank correlation test using SPSS17.0 software. A two-side $P$ value of $<0.05$ was considered statistically significant.

\section{Results}

There were no significant differences in the distribution of age or gender among the six groups of participants, and there were no significant differences in the serum HBVDNA levels among the five groups of chronic HBV infection (Table 1). 
Table 1 The demographic and clinical features of all the participants

\begin{tabular}{|c|c|c|c|c|c|c|}
\hline Parameters & CHB(ALT1-2ULN) & CHB(ALT2-5ULN) & CHB(ALT5-10ULN) & CHB(ALT $\geq 10 U L N)$ & CHBVIT & $\mathrm{HC}$ \\
\hline NO. & 30 & 30 & 30 & 30 & 20 & 28 \\
\hline \multicolumn{7}{|l|}{ Age (years) } \\
\hline Median (inter-quartile range) & $36.0(26.0-41.5)$ & $32.5(25.8-37.5)$ & $33.0(25.8-38.5)$ & $36.5(26.0-42.0)$ & $32.0(27.3-35.8)$ & $32.0(24.0-37.5)$ \\
\hline \multicolumn{7}{|l|}{$\operatorname{Sex} N(\%)$} \\
\hline Male & $18(60)$ & $20(67)$ & $21(70)$ & $19(64)$ & $12(67)$ & $18(64)$ \\
\hline Female & $12(40)$ & $10(33)$ & $9(30)$ & $11(36)$ & $8(33)$ & $10(36)$ \\
\hline \multicolumn{7}{|l|}{ HBVDNA (log 10 copies/mL) } \\
\hline Median (inter-quartile range) & $7.4(6.2-7.6)$ & $7.3(6.1-7.5)$ & $6.4(5.7-7.0)$ & $6.3(5.7-6.6)$ & $7.5(6.7-7.7)$ & NA \\
\hline HBeAg, pos/neg & $15 / 15$ & $16 / 14$ & $18 / 12$ & $16 / 14$ & $20 / 0$ & $0 / 28$ \\
\hline
\end{tabular}

Normal values: ALT: $1 \mathrm{ULN}=40 \mathrm{IU} / \mathrm{L}$. The lower limit of detection: HBVDNA $300 \mathrm{copies} / \mathrm{ml}$. Data were expressed as median and inter-quartile range or arithmetic mean and standard deviation (SD)

ALT alanine aminotransferase, $C H B$ chronic hepatitis $B, C H B V I T$ chronic hepatitis $B$ virus carriers in immuno-tolerant phase, $H B e A g$ hepatitis $B$ e antigen, $H B s A g$ hepatitis $B$ surface antigen, HBV DNA hepatitis B virus DNA; HC healthy control, ULN, upper limit of normal

The serum levels of ALT, IL-33 and ST2 in the six groups of participants were listed in Table 2 . The serum levels of IL-33 and ST2 were compared between each two out of those six groups. No significant difference was found in serum IL-33 levels between the CHB ALT12ULN group and the HC $(P=0.354)$. Other than that, there were significant differences when serum IL-33 levels were compared between any other two out of those six groups $(P<0.05$, respectively). Although the $P$ values were significant in above comparisons, the direction of the difference varied. IL-33 levels were significantly higher in healthy control group compared to the group in immunotolerant phase but it was significantly lower in healthy control group compared to the groups with ALT $>2$ times high (Fig. 1). In terms of serum ST2 concentrations, a similar trend was also observed. There were significant differences in serum ST2 levels between any other two out of those six groups $(P<0.05$, respectively), except for those between the CHB ALT1-2ULN group and the HC $(P=0.815)$. Although the $P$ values were significant in above comparisons, the direction of the difference varied. ST2 levels were significantly higher in healthy control group compared to the group in immunotolerant phase but it was significantly lower in healthy control group compared to the groups with ALT $>2$ times high (Fig. 2).
Next, the overall correlations between serum ALT and IL-33, as well as ST2 were analyzed. The result indicated that changes of serum IL-33 and ST2 levels were positively associated with ALT levels in patients with chronic HBV infection ( $r s=0.879, P<0.001$ for IL-33; $r s=0.923, P<0.001$ for ST2). However, there were no significant correlations between ALT and IL33 , or ST2 in healthy controls $(r s=-0.134, P=0.497$ for IL-33; $r s=-0.012, P=0.952$ for ST2) (Fig. 3).

Finally, when the levels of serum ALT [207 (84.5, $427.5)$ vs $182(76,460)]$, IL-33 [22.13 $(9.69,51.57)$ vs $18.13(8.76,45.7)]$ and ST2 $[41.78(12.49,147.20)$ vs $26.58(11.32,134.52)]$ were compared between the group with $\mathrm{HBeAg}$-positive chronic hepatitis $\mathrm{B}$ and the group with $\mathrm{HBeAg}$-negative chronic hepatitis $\mathrm{B}$, no significant differences were found $(P=0.635$ for ALT, $P=0.456$ for IL-33, and $P=0.413$ for ST2) (Fig. 4).

\section{Discussions}

The natural course of chronic HBV infection had been treated as being comprised of 4 phases: immune tolerance, immune clearance (HBeAg-positive chronic hepatitis B), inactive carrier state, and reactivation ( $\mathrm{HBeAg-negative}$ chronic hepatitis B). Understanding the dynamic nature of chronic HBV infection was crucial in the management of

Table 2 Serum levels of ALT, IL-33 and ST2

\begin{tabular}{lllll}
\hline Groups & NO. & ALT $(\mathrm{IU} / \mathrm{L})$ & $\mathrm{IL}-33(\mathrm{pg} / \mathrm{ml})$ & $\mathrm{ST2}(\mathrm{pg} / \mathrm{ml})$ \\
\hline HC & 28 & $26.0(23.3-31.5)$ & $9.6(6.6-12.7)$ & $10.6(6.5-13.6)$ \\
CHBVIT & 20 & $26.5(22.5-34.8)$ & $5.3(3.6-7.9)$ & $6.1(3.7-10.8)$ \\
CHB (ALT1-2ULN) & 30 & $57.5(49.0-69.8)$ & $7.8(4.5-10.9)$ & $9.9(6.2-13.2)$ \\
CHB (ALT2-5ULN) & 30 & $117.0(95.0-145.3)$ & $14.8(8.4-24.6)$ & $19.7(11.8-30.2)$ \\
CHB (ALT5-10ULN) & 30 & $268.0(237.5-319.5)$ & $29.9(11.8-21.8)$ & $80.9(33.6-69.7)$ \\
CHB (ALT $\geq 10 U L N)$ & 30 & $1005.0(637.0-1558.5)$ & $79.5(49.7-177.9)$ & $255.4(165.8-536.6)$ \\
\hline
\end{tabular}

Normal values: ALT: 1 ULN $=40 \mathrm{IU} / \mathrm{L}$. Data were expressed as median (inter-quartile range)

$A L T$ alanine aminotransferase, $C H B$ chronic hepatitis B, CHBVIT chronic hepatitis B virus carriers in immuno-tolerant phase, $H C$ healthy control, ULN upper limit of normal 


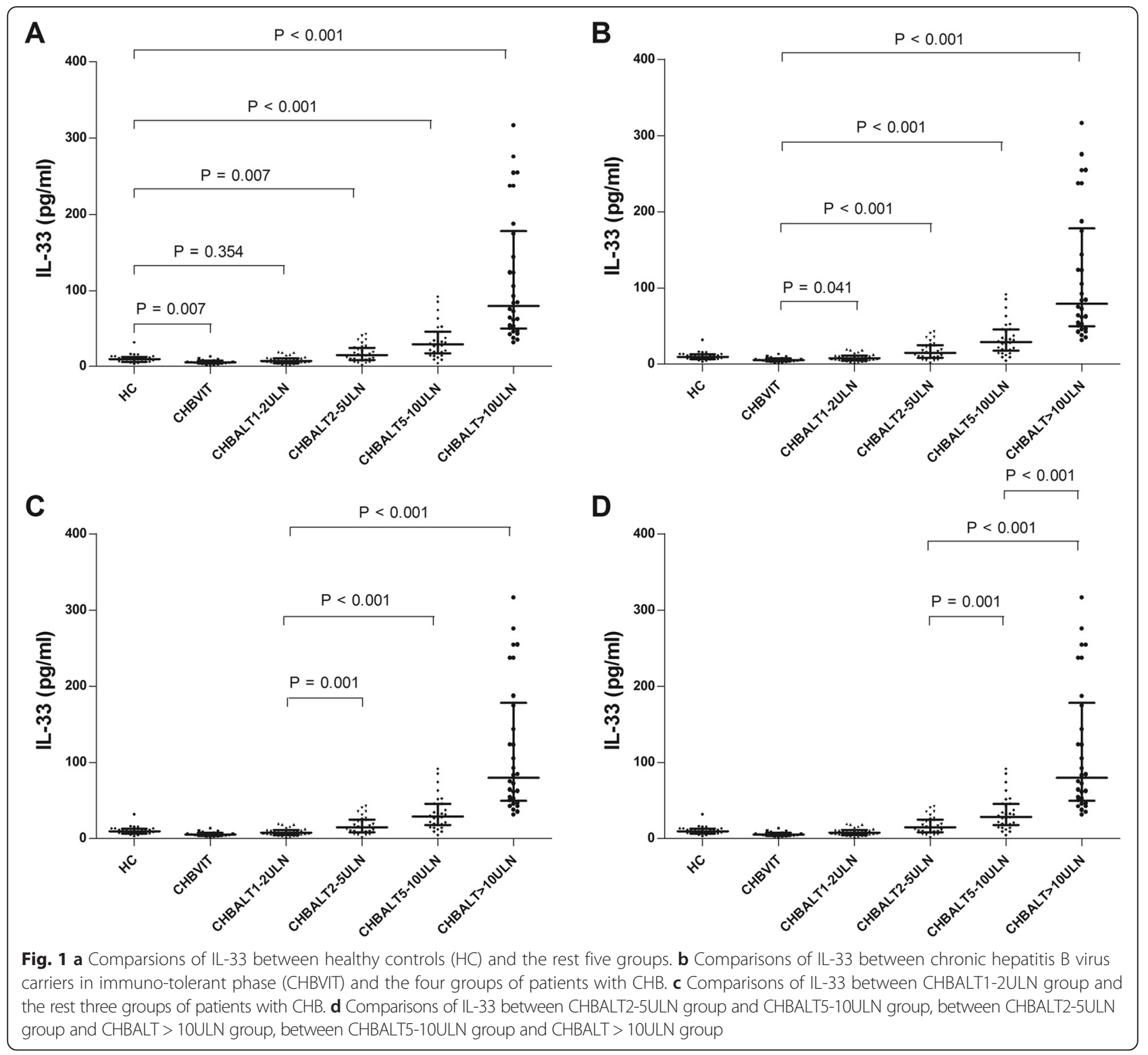

HBV carriers and underscored the need for long-term monitoring [13]. IL-33 was a member of the IL-1 family by classics appraisal. ST2 is a receptor for IL-33. Their multiple variants like ST2L or ST2V exist through alternative splicing. The main biological properties of IL-33 were to drive production of pro-inflammatory and Th2-associated cytokines in mast cells and Th2 lymphocytes [16, 17].

Aditionally, recent evidence suggested that IL-33 belonged to the larger family of damage-associated molecular pattern molecules and functioned as an "alarmin" similar to high-mobility-group-protein B1 (HMGB1) [16]. ST2 stimulated MYD88, IRAK1, IRAK4, and TRAF6 by phosphorylation of MAPK3/ERK1 and/or MAPK1/ ERK2, MAPK14, and MAPK8, Whereas ST2L exerted proinflammatory effects of IL-33. Increased serum levels of soluble ST2 had been reported in conditions like sepsis and dengue virus infection [18, 19]. Moreover, in murine inflammatory models, the production of pro-inflammatory cytokines preceded ST2 expression [20, 21]. Additionally, soluble ST2 could diminish the formation of inflammatory mediators [21, 22]. Hepatic over-expression of IL-33 had been detected in patients with liver fibrosis, chronic hepatitis B and hepatic failure. A previous study provided evidence for elevated levels of IL-33 and soluble ST2 in liver failure, which could be a sign of immune hyperactivation, and/or a mechanism to down-regulate inflammation. Especially, soluble ST2 may be useful to discern acute from chronic hepatic failure or to monitor the course and the severity of the disease [1]. Recent studies revealed that the levels of serum IL-33 were elevated in patients with 


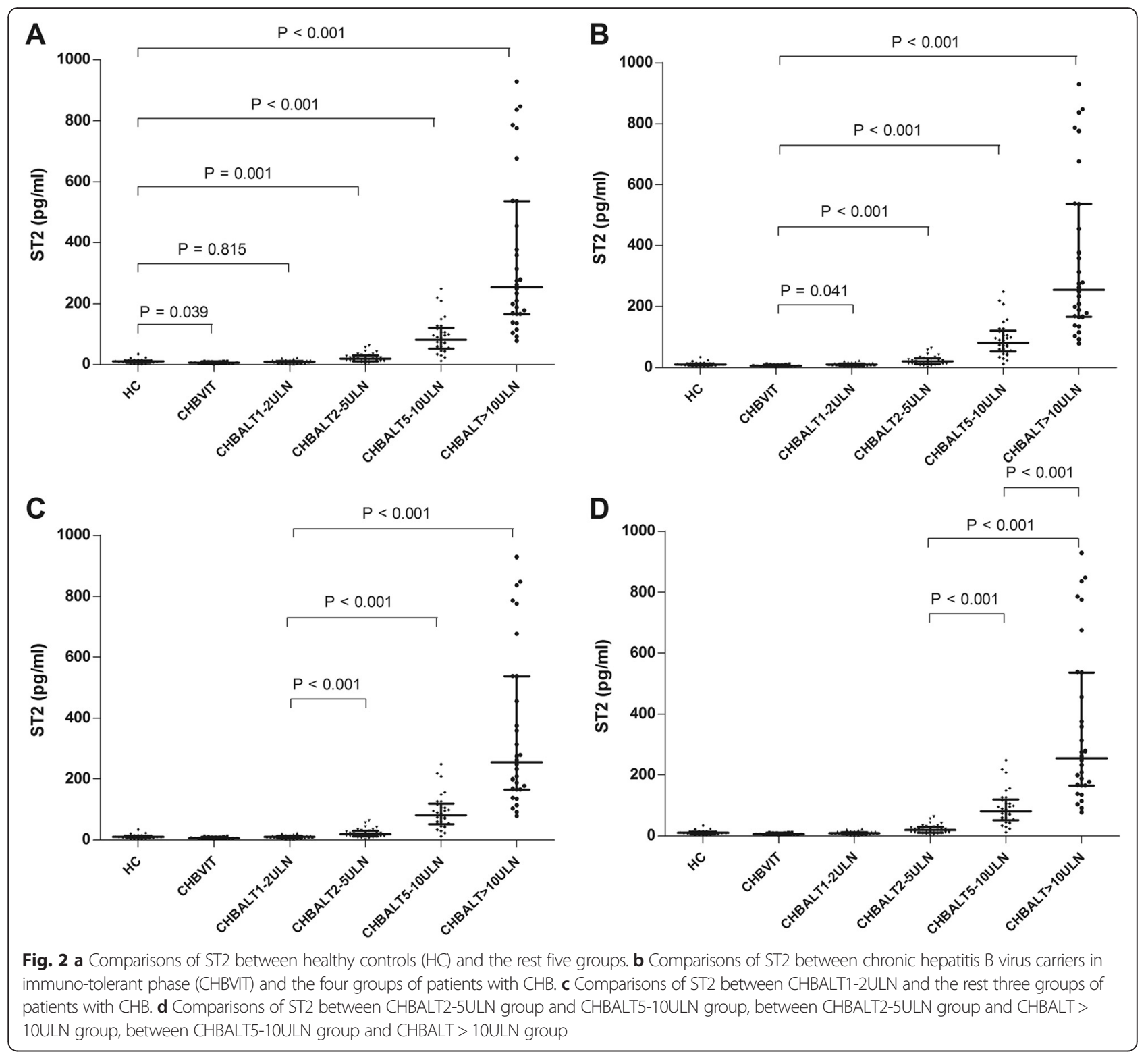

chronic hepatitis $\mathrm{C}$ and were dropped significantly after treatment with interferon, and Wang et al. found that there was a significant correlation between IL-33 and ALT concentration in chronic hepatitis $C[23,24]$. Other studies suggested that IL-33 participated in the pathogenic process of acute hepatitis induced by Con-A $[25,26]$ and IL-33 overexpression was associated with the development of $\mathrm{HBV} / \mathrm{HCV}$-related liver fibrosis [27]. Another study found that serum IL-33 levels in patients with CHB were significantly higher than those in healthy controls. Besides, treatment with adefovir dipivoxil to inhibit the replication of HBV dramatically decreased the levels of serum IL-33 in patients with $\mathrm{CHB}$. These confirmed that IL-33 could play a significant role in the progression of $\mathrm{CHB}$ and the data suggested that IL-33 might be a pathogenic factor in the pathogenic process of $\mathrm{CHB}$ patients, but no correlation was found between the levels of IL-33 and ALT or AST in their study. Further, serum ST2 levels were significantly higher in patients with $\mathrm{CHB}$ than those in healthy controls. Treatment with adefovir dipivoxil for 12 weeks did not significantly change the levels of serum ST2 [10]. A previous study had shown that serum ST2 levels in patients with acute liver failure were higher than those in patients with chronic liver failure and $\mathrm{HC}$ [1]. It was possible that high levels of serum ST2 were an early marker for liver injury, while high levels of serum IL-33 may be associated with the development and progression of liver fibrosis and damage [27]. Another study showed that the dendritic cells 

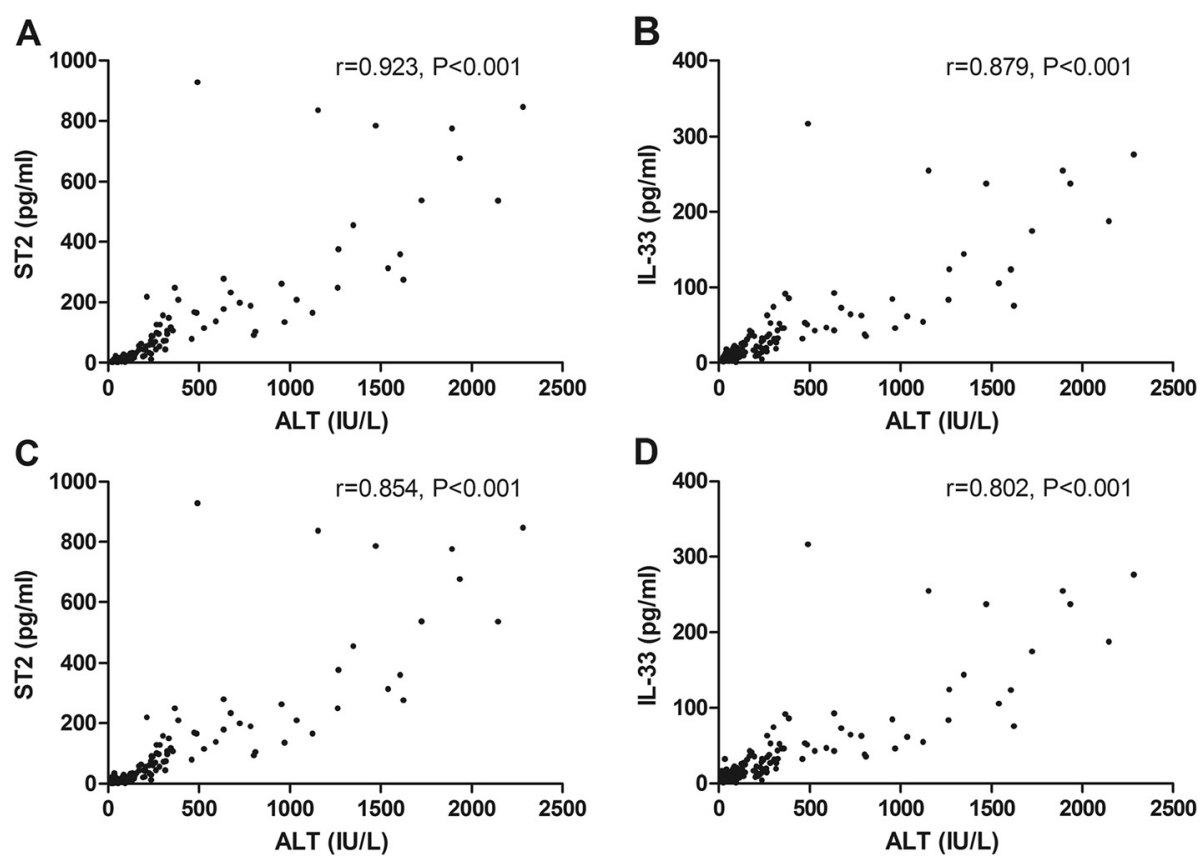

Fig. 3 a The overall correlation between ALT and IL-33 in patients with chronic HBV infection. $\mathbf{b}$ The overall correlation between ALT and ST2 in patients with chronic HBV infection. c The overall correlation between ALT and IL-33 in healthy controls. d The overall correlation between ALT and ST2 in healthy controls

responded directly to IL-33 through ST2. The IL-33 and DC interaction may represent a new pathway to initiate Th2-type immune responses [28].

Our study showed that the serum levels of IL-33 and ST2 of the chronic hepatitis B virus carriers in immunotolerant phase were significantly lower than those of the HC $(P=0.007$ for IL-33 and $P=0.039$ for ST2). The serum levels of IL-33 and ST2 in patients with CHB ALT1ULN-2ULN group were lower when compared with those in the $\mathrm{HC}$, however, the difference was not significant $(P=0.354$ for IL-33 and $P=0.815$ for ST2). The serum levels of IL-33 and ST2 in patients with CHB ALT $\geq 2 \mathrm{ULN}$ groups were significantly higher than those in the $\mathrm{HC}$, respectively $(P<0.05$, respectively). Changes of serum IL-33 and ST2 levels were found to be positively associated with ALT levels in patients with chronic HBV infection ( $r s=0.879, P<0.001$ for IL-33; $r s=0.923, P<0.001$ for ST2). However, there was no significant correlation between ALT and IL-33, or ST2 in healthy controls $(r s=-0.134, P=0.497$ for IL-33; $r s=-0.012, P=0.952$ for ST2). There were no significant differences in the serum levels of IL-33 and ST2 between patients with $\mathrm{HBeAg}$-positive $\mathrm{CHB}$ and those with $\mathrm{HBeAg-negative} \mathrm{CHB}$. Our data provided evidence that serum levels of IL-33 and ST2 elevated with the increase of ALT levels in patients with CHB, and reduced in patients with CHB ALT1-2ULN and chronic hepatitis B virus carriers in immunotolerant phase.

There were several limitations with our study. We did not carry out any multivariate analysis of all parameters that might associate with serum IL-33 and ST2 levels
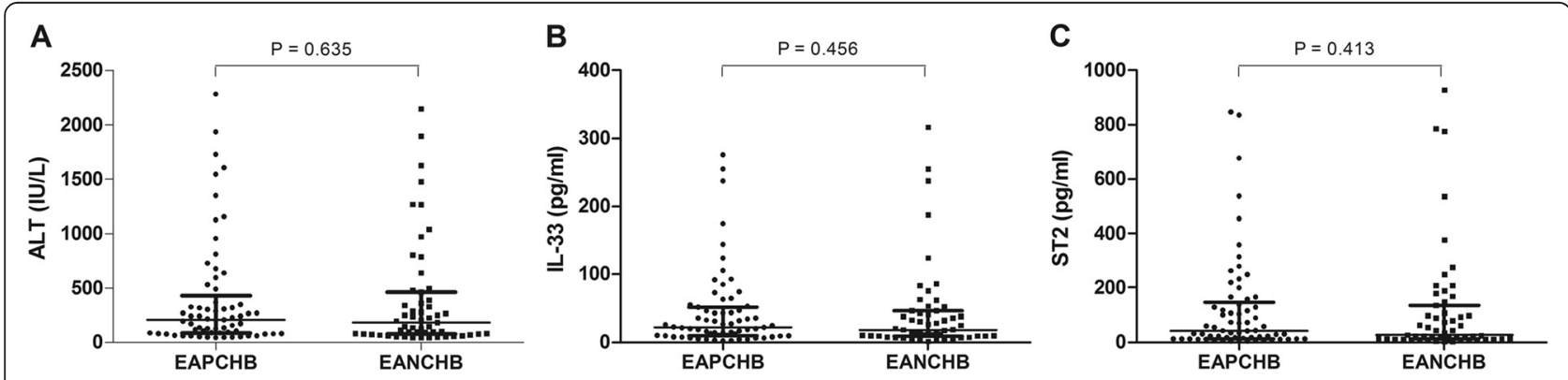

Fig. 4 a Comparisons of ALT between EAPCHB (HBeAg-positive chronic hepatitis B) group and EANCHB (HBeAg-negative chronic hepatitis B) group. $\mathbf{b}$ Comparisons of IL-33 between EAPCHB group and EANCHB group. $\mathbf{c}$ Comparisons of ST2 between EAPCHB group and EANCHB group 
due to the small sample size. Besides, we didn't conduct liver biopsy so that we did not figure out the source for IL-33 and ST2 through histopathological examinations. Although more detailed studies were necessary to determine the role and mechanisms of IL-33 and ST2 in the pathogenic process of chronic hepatitis B virus infection, our novel findings might provide new insights into understanding the role of IL-33 and ST2 in the pathogenesis of chronic hepatitis B virus infection. We speculated that IL-33 and ST2 could be used as an indicator to judge the patient's condition, which could help doctors choose antiviral drugs for the patients and assess the therapeutic effects. We will conduct further study to investigate the changes of serum IL-33 and ST2 after antiviral treatment in future.

\section{Conclusions}

In conclusion, our data indicated that serum levels of IL-33 and ST2 elevated with the increase of ALT levels in patients with CHB. The serum levels of IL-33 and ST2 varied in different courses of chronic hepatitis B virus infection. We surmised that IL-33 and ST2 might indicate liver damage of patients with chronic hepatitis $B$, just like ALT. They were associated with the natural course of chronic hepatitis B virus infection.

\section{Availability of data and materials}

The raw data will be provided upon request by Dr. Kai Wang (Correspondence author), Email: wangdoc876@126.com; wangdoc2010@163.com.

\section{Abbreviations}

ALT: alanine aminotransferase; CHB: chronic hepatitis B; ELISA: enzyme-linked immunosorbent assay; HBV: hepatitis B virus; HC: healthy control; IL33: Interleukin-33; PCR: polymerase chain reaction; Th2: T helper-2; ULN: upper limit of normal.

\section{Competing interests}

The authors declare that they have no competing interests.

\section{Authors' contributions}

SLH was involved in drafting the manuscript and acquisition of data; JGZ completed analysis and interpretation of data; ZLW helped the acquisition and analysis of data; SG contributed to the statistical analysis and correction of the manuscript. KW designed this study and gave final approval of the version to be published. All authors have read and approved the final version of the manuscript.

\section{Acknowledgments}

This study was funded by grants from Key Project of Chinese Ministry of Science and Technology (2012ZX10002007, 2013ZX10002001), National Natural Science Foundation of China (81171579, 81201287), Natural Science Foundation of Shandong Province (ZR2010HM070, ZR2010HQ040), and Science and Technology Development Plan of Shandong Province (2014GSF118068)

\section{Author details}

'Department of Hepatology, Qilu Hospital of Shandong University, Jinan 250012, Shandong, China. ${ }^{2}$ Department of clinical laboratory, Qingdao Municipal Infectious Disease Hospital, Qingdao 266033, Shandong, China. ${ }^{3}$ Institute of Hepatology, Shandong University, Wenhuaxi Road 107\#, Jinan 250012, Shandong, China.
Received: 24 November 2014 Accepted: 6 May 2016

Published online: 16 May 2016

\section{References}

1. Roth GA, Zimmermann M, Lubsczyk BA, et al. Up-regulation of interleukin 33 and soluble ST2 serum levels in liver failure. J Surg Res. 2010;163:e79-83.

2. Chen J, Yuan Z. Interplay between hepatitis B virus and the innate immune responses: implications for new therapeutic strategies. Virol Sin. 2014;29:17-24.

3. Crockett SD, Keeffe EB. Natural history and treatment of hepatitis B virus and hepatitis C virus coinfection. Ann Clin Microbiol Antimicrob. 2005:4:13.

4. Rehermann B, Nascimbeni M. Immunology of hepatitis B virus and hepatitis C virus infection. Nat Rev Immunol. 2005:5:215-29.

5. Rehermann B. Interaction between the hepatitis $C$ virus and the immune system. Semin Liver Dis. 2000;20:127-41.

6. Gonzalez MI, Rubinstein N, llarregui JM, Toscano MA, Sanjuan NA, Rabinovich GA. Regulated expression of galectin-1 after in vitro productive infection with herpes simplex virus type 1: implications for T cell apoptosis. Int J Immunopathol Pharmacol. 2005;18:615-23.

7. Martini F, Agrati C, D'Offizi G, Poccia F. HLA-E up-regulation induced by HIV infection may directly contribute to CD94-mediated impairment of NK cells. Int J Immunopathol Pharmacol. 2005;18:269-76.

8. Falasca K, Ucciferri C, Dalessandro M, et al. Cytokine patterns correlate with liver damage in patients with chronic hepatitis B and C. Ann Clin Lab Sci. 2006;36:144-50

9. Grzegorzewska AE, Wobszal P, Jagodzinski PP. Interleukin-18 promoter polymorphism and development of antibodies to surface antigen of hepatitis B virus in hemodialysis patients. Kidney Blood Press Res. 2012;35:1-8

10. Wang J, Cai Y, Ji H, et al. Serum IL-33 levels are associated with liver damage in patients with chronic hepatitis B. J Interferon Cytokine Res. 2012;32:248-53.

11. Bertoletti A, Ferrari C. Innate and adaptive immune responses in chronic hepatitis B virus infections: towards restoration of immune control of viral infection. Gut. 2012;61:1754-64.

12. Chang J, Block TM, Guo JT. The innate immune response to hepatitis B virus infection: implications for pathogenesis and therapy. Antiviral Res. 2012;96:405-13

13. Yim HJ, Lok AS. Natural history of chronic hepatitis B virus infection: what we knew in 1981 and what we know in 2005. Hepatology. 2006:43:S173-81.

14. Liaw YF, Kao JH, Piratvisuth $T$, et al. Asian-Pacific consensus statement on the management of chronic hepatitis B: a 2012 update. Hepatol Int. 2012:6:531-61.

15. Jiang $Y, M a Z$, Xin $G$, et al. Th1 and Th2 immune response in chronic hepatitis B patients during a long-term treatment with adefovir dipivoxil. Mediators Inflamm. 2010;2010:143026.

16. Moussion C, Ortega N, Girard JP. The IL-1-like cytokine IL-33 is constitutively expressed in the nucleus of endothelial cells and epithelial cells in vivo: a novel 'alarmin'? PLoS One. 2008;3, e3331.

17. Schmitz J, Owyang A, Oldham E, et al. IL-33, an interleukin-1-like cytokine that signals via the IL-1 receptor-related protein ST2 and induces T helper type 2-associated cytokines. Immunity. 2005;23:479-90.

18. Brunner M, Krenn C, Roth $G$, et al. Increased levels of soluble ST2 protein and IgG1 production in patients with sepsis and trauma. Intensive Care Med. 2004;30:1468-73.

19. Becerra A, Warke RV, de Bosch N, Rothman AL, Bosch I. Elevated levels of soluble ST2 protein in dengue virus infected patients. Cytokine. 2008:41:114-20

20. Kumar S, Tzimas MN, Griswold DE, Young PR. Expression of ST2, an interleukin-1 receptor homologue, is induced by proinflammatory stimuli. Biochem Biophys Res Commun. 1997;235:474-8.

21. Oshikawa K, Yanagisawa K, Tominaga S, Sugiyama Y. ST2 protein induced by inflammatory stimuli can modulate acute lung inflammation. Biochem Biophys Res Commun. 2002;299:18-24.

22. Leung BP, Xu D, Culshaw S, Mclnnes IB, Liew FY. A novel therapy of murine collagen-induced arthritis with soluble T1/ST2. J Immunol. 2004;173:145-50.

23. Cacopardo B, Rita Pinzone M, Palermo F, Nunnari G. Changes in serum Interleukin-33 concentration before and after treatment with pegylated interferon alfa-2a plus ribavirin in patients with chronic hepatitis C genotype 1b infection. Hepat Mon. 2012;12, e7611. 
24. Wang J, Zhao P, Guo H, et al. Serum IL-33 levels are associated with liver damage in patients with chronic hepatitis C. Mediators Inflamm. 2012;2012:819636.

25. Arshad MI, Rauch M, L'Helgoualc'h A, et al. NKT cells are required to induce high IL-33 expression in hepatocytes during ConA-induced acute hepatitis. Eur J Immunol. 2011:41:2341-8.

26. Volarevic V, Mitrovic M, Milovanovic M, et al. Protective role of IL-33/ST2 axis in Con A-induced hepatitis. J Hepatol. 2012;56:26-33.

27. Marvie $P$, Lisbonne M, L'Helgoualc'h $A$, et al. Interleukin-33 overexpression is associated with liver fibrosis in mice and humans. J Cell Mol Med. 2010:14:1726-39.

28. Rank MA, Kobayashi T, Kozaki H, Bartemes KR, Squillace DL, Kita H. IL-33activated dendritic cells induce an atypical TH2-type response. J Allergy Clin Immunol. 2009;123:1047-54.

Submit your next manuscript to BioMed Central and we will help you at every step:

- We accept pre-submission inquiries

- Our selector tool helps you to find the most relevant journal

- We provide round the clock customer support

- Convenient online submission

- Thorough peer review

- Inclusion in PubMed and all major indexing services

- Maximum visibility for your research

Submit your manuscript at www.biomedcentral.com/submit
Biomed Central 